\title{
Photogenerated carrier profile determination in polymeric light-emitting diodes by steady-state and transient photocurrent measurements
}

\author{
L.F. Santos ${ }^{\text {a }}$, R.F. Bianchi ${ }^{\text {a }}$, R.M. Faria ${ }^{a}$, S. Mergulhão ${ }^{b, *}$ \\ a Instituto de Física de São Carlos, Universidade de São Paulo, CP 369, 13560-970 São Carlos, SP, Brazil \\ ${ }^{\mathrm{b}}$ Departamento de Física, Universidade Federal de São Carlos, CP 676, 13565-905 São Carlos, SP, Brazil
}

Received 29 July 2005; received in revised form 27 March 2006; accepted 17 August 2006

\begin{abstract}
Photogenerated charge carrier profiles in poly(2-methoxy-5-(2'-ethyl-hexyloxy)-1,4-phenylene vinylene) (MEH-PPV) light-emitting diodes were determined from steady-state photocurrent spectra and transient photocurrent decay measurements. The observation that the photocurrent spectra behavior is strongly dependent on the bias polarity and amplitude suggests the existence of an intrinsic electric field, determined by the difference in the work function of the metallic electrodes, as well as a field dependence on the free charge carrier generation rates. The obtained results reveal built-in voltages of +0.2 and $-0.6 \mathrm{~V}$ for $\mathrm{Au}$ and $\mathrm{Al}$ electrodes, respectively. The photocurrent spectra can be semi-quantitatively explained using a simple model which takes into account the internal built-in electric field of the device structure, the band gap energy and the migration/diffusion of the photogenerated charge carriers through the polymeric film. The drift mobility of carriers was investigated by the time-of-flight technique (TOF). The value for the mobility of holes was obtained from the change of slopes in the double logarithmic plot of the transient current and was independent of the applied field. The TOF transients for electrons showed no change of slopes in the double logarithmic plot and the drift mobility of electrons could not be calculated.
\end{abstract}

(C) 2006 Elsevier B.V. All rights reserved.

Keywords: Polymers; Photoconduction; Mobility

\section{Introduction}

Conjugated polymers have attracted much attention in the last 15 years due to their semiconducting properties and to their potential application in a wide variety of electronic and optoelectronic devices like light-emitting diodes, photovoltaic cells and field-effect transistors. However, despite the great advance in the development and improvement of new materials and structures, there are still several fundamental aspects related to the excited states in conjugated polymers that are not yet completely understood. One of the most controversial topics discussed in the physics of conjugated polymers is about the nature of the excited states in such materials. In this sense, two opposite positions are generally adopted: one is the traditional semiconductor band model and the other one is based on the formation of bound excited states due to the molecu-

\footnotetext{
* Correspondence to: R Luiz Roher, 355, São Carlos, CEP 13570-002, SP, Brazil. Tel.: +55 163351 8223; fax: +55 1633614835 .

E-mail address: dsme@df.ufscar.br (S. Mergulhão).
}

lar character of organic materials. For this reason, photocurrent spectroscopy is a powerful techinique used to study charge carrier photogeneration and transport in semiconductor devices. Since the photo-excitation generates charged states, which can give rise to a measurable photoconductivity if mobile charge carriers are produced, the spectral dependence of the photocurrent is frequently used to argue in favor of the band or the excitonic model. In organic molecular solids and highly crystalline conjugated polymers, there is a large energy difference between the onset of the optical absorption and the onset of the photoconductivity, which is unquestionably accepted as evidence of the influence of electron-electron interactions in these materials. On the other hand, poly ( $p$-phenylene vinylene)'s (PPV's) usually present onsets of the photoconductivity and the absorption which are practically coincident [1]. This has been cited as evidence of a semiconducting band model [2-5], with direct photogeneration of free positive and negative carriers, although charge generation is commonly considered as a secondary process, resulting from dissociation of excitons by interaction with defects and impurities in the vicinities of interfaces. Other authors, however, assign this behavior to additional factors $[6,7]$ to develop 
semi-quantitative models particularly based on the sharp rise of the photocurrent which is usually observed at the low energy tail of the absorption spectrum [8]. In this communication, we present experimental results based on steady-state and transient photocurrent measurements in order to determine the free carrier photogeneration profile in polymeric light-emitting devices based on PPV derivatives. This study can give quantitative as well as qualitative information about the nature of conjugated polymers.

\section{Experimental details}

The alkoxy-substituted PPV derivative poly(2-methoxy-5(2'-ethyl-hexyloxy)-1,4-phenylene vinylene) (MEH-PPV) was synthesized using a modified Gilch route as described in detail elsewhere [9]. The average molecular weight of the obtained orange-reddish polymer was $69.000 \mathrm{~g} \mathrm{~mol}^{-1}$ and the poly-dispersity was about six. The devices were produced by initially etching $14 \mathrm{~mm} \times 12 \mathrm{~mm}$ ITO-coated glass substrates to give rise to properly designed transparent electrodes. The ITO substrates were further cleaned by rinsing in acetone, rubbing with isopropanol and sonication in dilute aqua regia $\left(\mathrm{HNO}_{2}+\mathrm{HCl}\right)$ solution for $10 \mathrm{~min}$. After abundantly washing the wafers in de-ionized water and drying with pure nitrogen, MEH-PPV films (400-800 nm thick) were obtained by spin-casting a $10 \mathrm{mg} \mathrm{mL}^{-1}$ chloroform solution onto the substrates. The films were dried in a vacuum at $80^{\circ} \mathrm{C}$ for $4 \mathrm{~h}$ before vacuum thermal evaporation of the metallic top electrodes. Two different non-transparent metallic electrodes $(30 \mathrm{~nm}$ of $\mathrm{Au}$ and $50 \mathrm{~nm}$ of $\mathrm{Al}$ ) with $8 \mathrm{~mm}^{2}$ in area were deposited side by side, producing two distinct devices with similar geometric characteristics.

The photocurrent measurements were performed with the devices placed in a nitrogen gas flow cryostat, with transparent quartz windows, to avoid oxidation of the sample. Selective wavelength photo-excitation was performed by focusing onto the sample the light output from a monochromator (Oriel Instruments) used to separate the spectral response of a $450 \mathrm{~W}$ Xe lamp. Scanning of the incident light wavelength in the $300-800 \mathrm{~nm}$ range was carried out at rates varying from 24 up to $120 \mathrm{~nm} \mathrm{~min}^{-1}$. Since no variations in the photocurrent spectra were observed in the used scanning rates, the charge separation and transport processes were considered as fast enough to not interfere in the spectra at the observed time scale. The sample short-circuit current was monitored by an electrometer (Keithley 6517A) which was also used to provide the external applied bias. The lamp spectrum was recorded by a UV-vis sensitive photodiode, simultaneously with a current sampling the monochromatic light through a beam splitter (as schematically shown in Fig. 1a). All photocurrent spectra were corrected from the measured lamp spectrum, the photodiode response and the ITO substrate absorption curve, considering that the measured radiant flux (at $400 \mathrm{~nm}$ ) in the sample was $0.65 \mathrm{~mW} \mathrm{~cm}^{-2}$. Fig. 1b represents the device structure during the photocurrent measurement. The monochromatic light passes through the ITO electrode before reaching the polymeric film. In despite of the electrical connections shown in Fig. 1b, the forward direction, in the framework of this communication, is determined when the ITO electrode is positively polarized. For the transient current measurements, a pulsed nitrogen laser (Oriel Instruments) was used to generate short pulses ( $5 \mathrm{~ns}$ length) to excite the samples through the ITO electrode. A Coumarine 503 Oriel Dye was used to excite the sample with a $490 \mathrm{~nm}$ laser. The current transients were measured using a broadband current amplifier (Stanford Research SR570) coupled to a fast digital oscilloscope (Tektronix $340 \mathrm{~A}$ ), triggered by the laser pulse.

\section{Results and discussion}

The photocurrent action spectra of ITO/MEH-PPV/Al and ITO/MEH-PPV/Au devices are shown in Fig. 2. The upper graphs show the normalized photocurrent spectra, in comparison with the absorption spectrum of MEH-PPV. The response of the photocurrent action spectrum is usually classified as being of two types: symbatic, if the photocurrent spectrum follows the absorption spectrum and antibatic if the photocurrent spectrum behaves differently of the absorption spectrum, presenting almost complementary characters [10]. In Fig. 2a, the ITO/MEH-PPV/Al device shows a clear antibatic behavior when short-circuited or negatively biased $\left(V_{\mathrm{b}}=-1.5 \mathrm{~V}\right)$, characterized by a evident sharp peak around $2.2 \mathrm{eV}$ and practically null photocurrent values in the region where the MEH-PPV strongly absorbs, between 2.3 and $2.9 \mathrm{eV}$. On the other hand, for the device biased positively $\left(V_{\mathrm{b}}=+1.5 \mathrm{~V}\right)$, the photocurrent spectrum visibly switches to a symbatic behavior. For photon energies above $3 \mathrm{eV}$, the photocurrent increases continuously to the energy upper limit

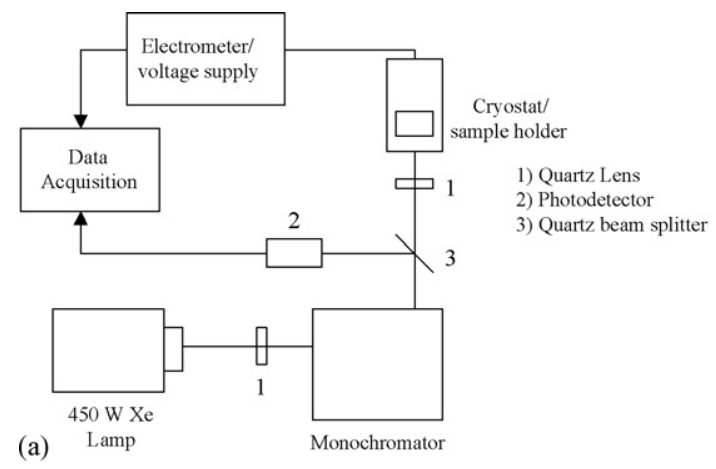

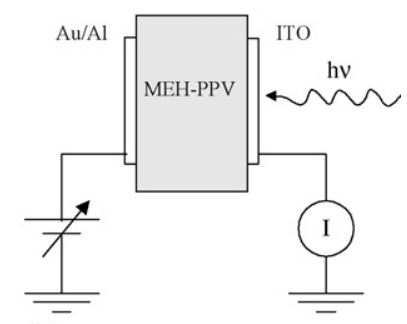

(b)

Fig. 1. (a) Schematics of the experimental setup used in the photocurrent measurements and (b) illustration of the device structure during the experiments. 

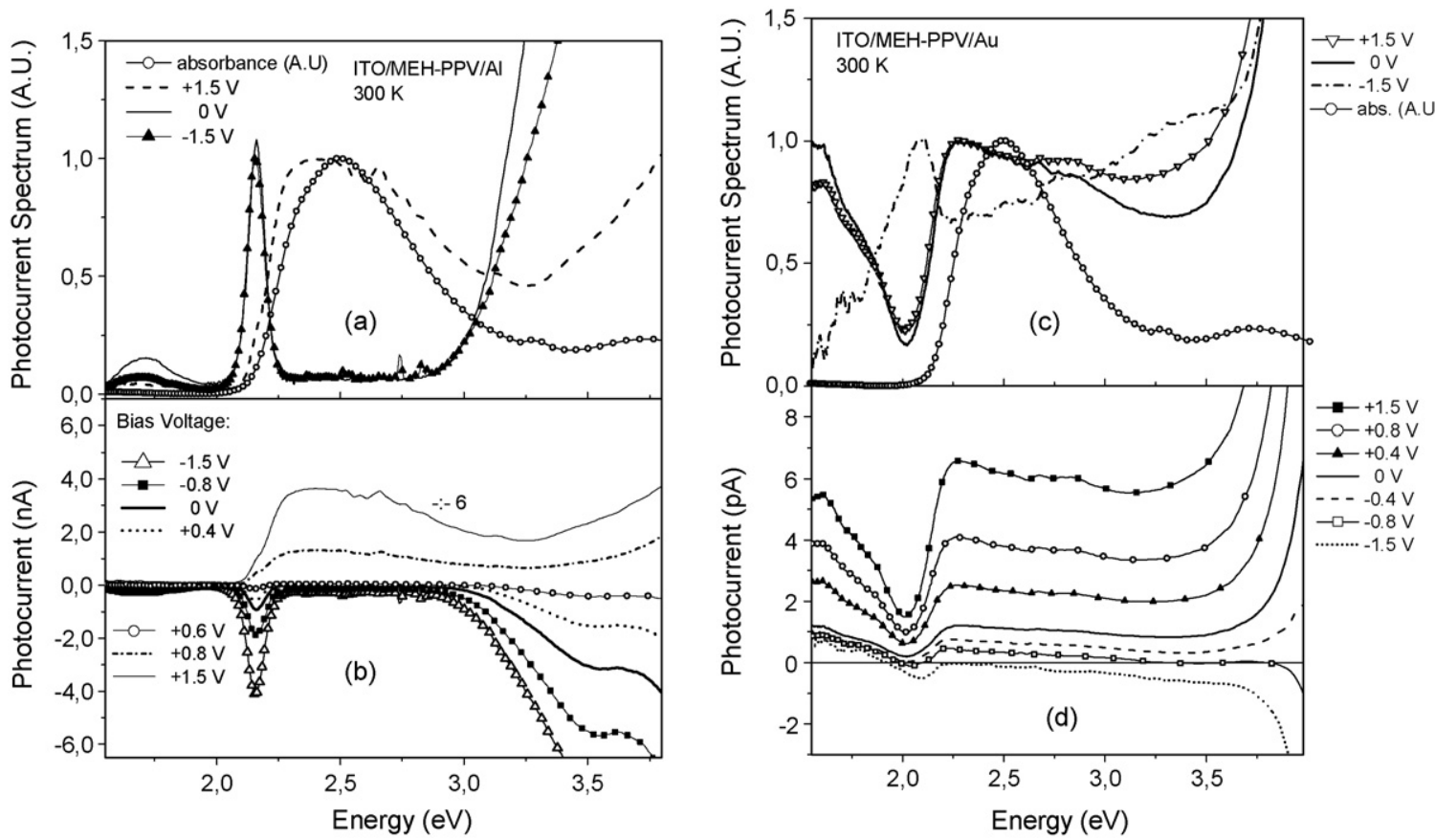

Fig. 2. Photocurrent spectra of a ITO/MEH-PPV/Al device (a) normalized spectrum, (b) absolute values and a ITO/MEH-PPV/Au device (c) normalized spectrum and (d) absolute values at $300 \mathrm{~K}$ for different bias voltages.

attainable by the experimental setup $(4.1 \mathrm{eV})$, regardless the bias polarity.

Fig. $2 \mathrm{~b}$ shows the voltage dependence of the photocurrent spectra of the ITO/MEH-PPV/Al device. For negative voltages, the photocurrent, which always presents a negative value, increases in amplitude with the bias. For positive voltages, the photocurrent still presents negative values, decreasing in amplitude with increasing bias from 0 to $+0.6 \mathrm{~V}$. Above this voltage, the photocurrent curves switch the polarity and start to present the symbatic behavior described before. Moreover, the onset of the photocurrent in the high energy regime is slightly higher $(\sim 3.5 \mathrm{eV})$ than observed for negative biases $(\sim 3.1 \mathrm{eV})$.

For the ITO/MEH-PPV/Au device (Fig. 2c and d) the photocurrent values are almost three orders of magnitude lower than those observed for the ITO/MEH-PPV/Al device. For the device biased positively, the absolute photocurrent decreases from low to high energies, reaching a minimum around $2 \mathrm{eV}$. For energies higher than $2.2 \mathrm{eV}$, the photocurrent starts to increase, showing a symbatic behavior in the region where MEH-PPV absorbs well. For energies above $3.5 \mathrm{eV}$, the photocurrent increases steeply, as already observed for the ITO/MEH-PPV/Al device. For negative voltages, the photocurrent spectrum is practically antibatic in the whole energy range. Differently of the ITO/MEH-PPV/Al device, the device with gold cathode has a short-circuit photocurrent spectrum similar to those obtained with the device biased positively. For negative bias until $-0.4 \mathrm{~V}$, the spectrum is similar as for short-circuit showing, however, lower photocurrent values. For higher voltages, the minimum in the photocurrent at $2.0 \mathrm{eV}$ still persists but the photocurrent switches to negative values.

The observed results indicate the formation of internal builtin electric fields in both kind of devices. Since the short- circuit photocurrents present contrary behaviors (negative for the ITO/MEH-PPV/Al and positive for the ITO/MEH-PPV/Au), these electric fields must have opposite directions. These builtin voltages can be roughly estimated by the difference between the ITO and the metallic electrodes work-functions. For the ITO/MEH-PPV/Al device, this voltage $\left(V_{\mathrm{b}}=\left(\phi_{\mathrm{Al}}-\phi_{\mathrm{ITO}}\right) / e\right)$ should vary from -0.3 to $-0.7 \mathrm{~V}\left(\phi_{\text {ITO }}=4.6-4.8 \mathrm{eV}\right.$ and $\phi_{\mathrm{Al}}=4.1-4.3 \mathrm{eV}$ ) whereas, for the ITO/MEH-PPV/Au device, it should be between +0.2 and $+0.6 \mathrm{~V}\left(\phi_{\mathrm{Au}}=5.0-5.2 \mathrm{eV}\right)$.

The built-in voltage can be better observed analyzing the dc current-voltage $(I-V)$ curves obtained at different wavelengths as shown in Figs. 3 and 4.

As commonly observed in such structures, the $\mathrm{Al}$ device in the dark presents a rectifying behavior due to the expected better hole injection from ITO into the polymer. Under illumination at energies which do not present strong photocurrent spectrum (Fig. 3b), little variation occurs in the $I-V$ curve. For energies which present higher photocurrent spectra (Fig. 3a), a clear photovoltaic effect can be observed. Although the short-circuit current $\left(I_{\mathrm{sc}}\right)$ varies with the photon energy, the open-circuit voltage $\left(V_{\text {oc }}\right)$ is almost energy independent (about $\left.+0.6 \mathrm{~V}\right)$. The open-circuit voltage can be interpreted as the voltage required to overcome the built-in voltage $\left(V_{\mathrm{b}}\right)$ which, in this case, is $-0.6 \mathrm{~V}$, in agreement with the expected value.

The $I-V$ curves under different photon energies for the $\mathrm{Au}$ device are shown in Fig. 4. It can be observed that the photovoltaic effect is much less evident than in the Al device. At first sight, only an increase in the photoconductivity is observed at higher photon energies. However, small short-circuit currents and open-circuit voltages can also be observed (inset of Fig. 4). The highest value for $V_{\mathrm{oc}}$ was about $-0.2 \mathrm{~V}$, which indicates a built-in voltage of $+0.2 \mathrm{~V}$, as expected. 


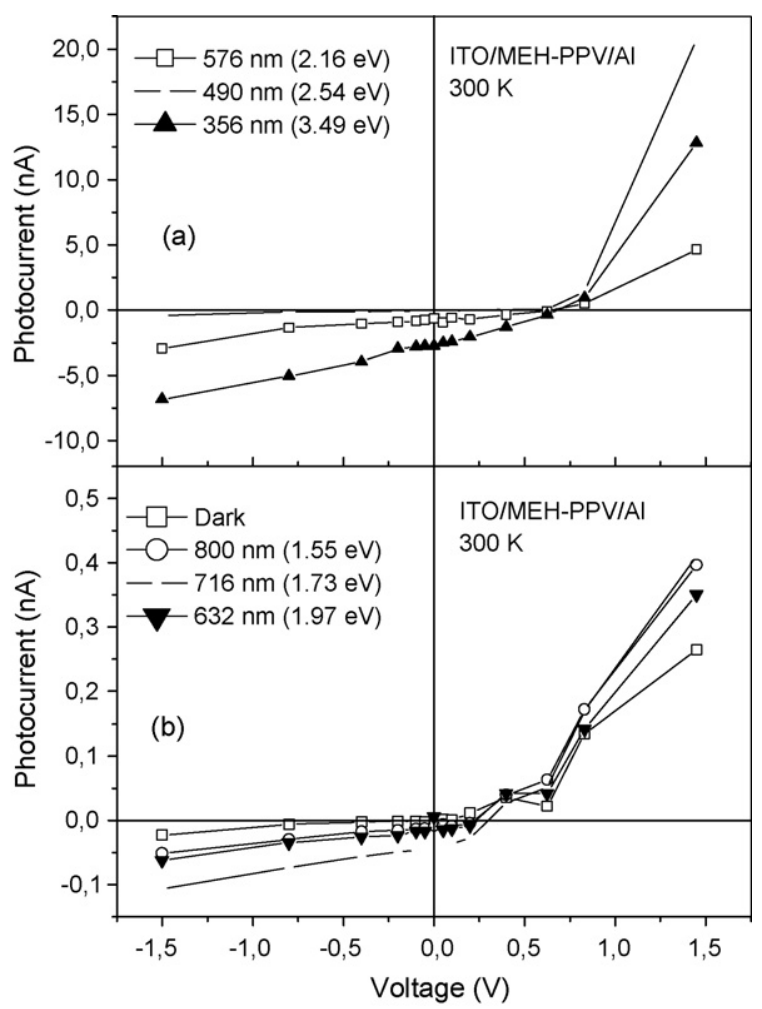

Fig. 3. $I-V$ curves for the ITO/MEH-PPV/Al device at $300 \mathrm{~K}$ for different photon energies (incident flux: $0.65 \mathrm{~mW} / \mathrm{cm}^{2}$ ): (a) $2.16,2.54$ and $3.49 \mathrm{eV}$ and (b) 1.55 , 1.73 and $1.97 \mathrm{eV}$.

In order to explain the symbatic/antibatic behavior of the photocurrent spectra, one can initially use a qualitative explanation based on the charge carrier generation profile, determined by the absorption spectrum, and the transport/diffusion of the free carriers towards the bulk. An appropriate way to determine the nature and the mobility of the major charge carriers is using the time-of-flight technique [11], which is based on the measurement of current transients due to fast light pulses.

Fig. 5 shows the current transients for the ITO/MEH-PPV/Al device (550 nm thick) for forward (Fig. 5a) and reverse (Fig. 5b)

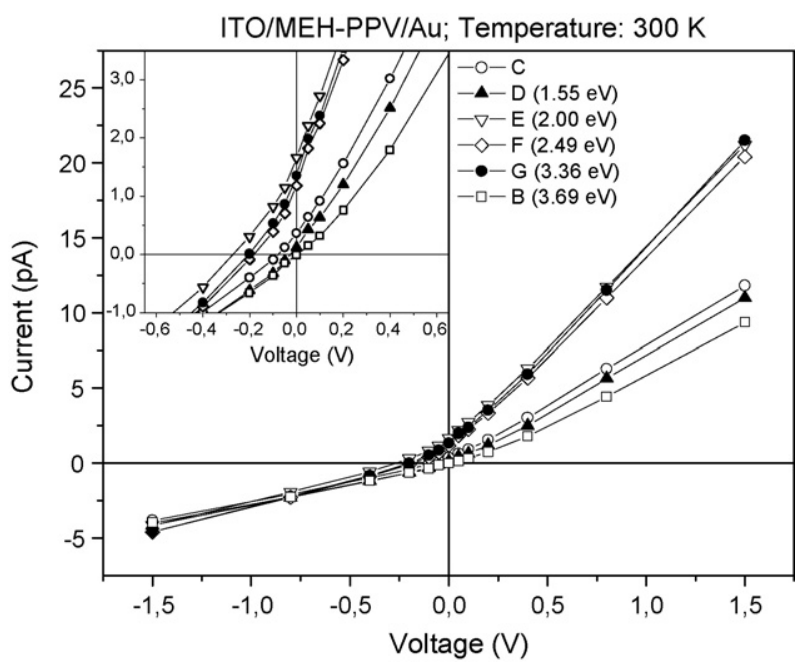

Fig. 4. I- $V$ characteristics on a ITO/MEH-PPV/Au device for different photon energies (incident flux: $0.65 \mathrm{~mW} / \mathrm{cm}^{2}$ ). applied bias. A cuvette with Coumarine 503 Oriel Dye was used to tune the laser wavelength $(337.1 \mathrm{~nm})$ to $490 \mathrm{~nm}$ ( $20 \mathrm{~nm}$ wide). The efficiency of the Dye is $19 \%$ and the injected charge was approximately $0.01 \mathrm{CV}$. This attenuation avoided the formation of space charge during the TOF experiment, and the electric field is not affected by the carriers photogenerated. The photocurrent is approximately two orders of magnitude higher than the dark current, so the transient photocurrent is not affected by the dark current. Since the absorption coefficient at the excitation wavelength is about $2 \times 10^{5} \mathrm{~cm}^{-1}$, for the used device thickness, most of the charge carriers are photogenerated close to the illuminated surface (approximately $9 \%$ of sample thickness). When the ITO electrode is biased positively, the superficial positive photogenerated free charge carriers move towards the bulk whereas the negative carriers should be predominantly collected by the front electrode. The transient photocurrent is dominated by holes, but the "effective thickness" of the sample is about $90 \%$ of the sample thickness, so, the mobility may be under estimated. The expected error in the mobility is $10 \%$. At low bias values (below $+4 \mathrm{~V}$ ), the transit time of the charge carriers is too long to be observed in the used time scale, originating long-lasting current decays. For higher biases, the transit time of the carrier can be determined by the change in the slope of the current transient in the log-log plot (Fig. 5a). The calculated hole mobility for this sample is field independent and its mean value is about $4 \times 10^{-6} \mathrm{~cm}^{2} /(\mathrm{V} \mathrm{s})$. For the ITO polarized negatively, the current transients are expected to be due to negative free charge carriers. In the whole applied bias range (0 to $-10 \mathrm{~V})$, no transition in the $\log -\log$ plot of the current transients could be observed, only the same long current decays observed at low positive voltages. This indicates that the electron mobility is much lower (at least two orders of magnitude) than the hole mobility in MEH-PPV.

Taking into account the information that the negative charge carriers are much less mobile than the positive ones, the short-circuit photocurrent spectrum behavior for the ITO/MEHPPV/Al device can be understood by the photogeneration profile and the respective transport of the carriers into the bulk. At high wavelengths (low energies), the photon energy is not sufficient to overcome the polymer gap (about $2.2 \mathrm{eV}$ ) and practically no photocurrent values are observed. As the photon energy becomes closer to the absorption excitonic band edge, bulk charge generation occurs and the built-in voltage, predominantly determined by the $\mathrm{Al} /$ polymer interface, makes the free carriers drift towards the ITO electrode, leading to negative current values. As the absorption coefficient increases, less free carriers are photogenerated at the $\mathrm{Al} /$ polymer interface and the built-in voltage begins to be dominated by the ITO/polymer interface, which makes negative charge carriers go into the bulk. Since negative carriers are less mobile and the contact potential at the ITO/polymer interface is expected to be much lower than at the $\mathrm{Al} /$ polymer interface, the photocurrent assumes again low values. As the excitation wavelength gets out of the excitonic band absorption, charge carriers start to be again photogenerated at the $\mathrm{Al} /$ polymer interface, giving rise to the observed increase in the photocurrent at high energies. This explanation is also valid when the device is biased negatively. When sufficiently 

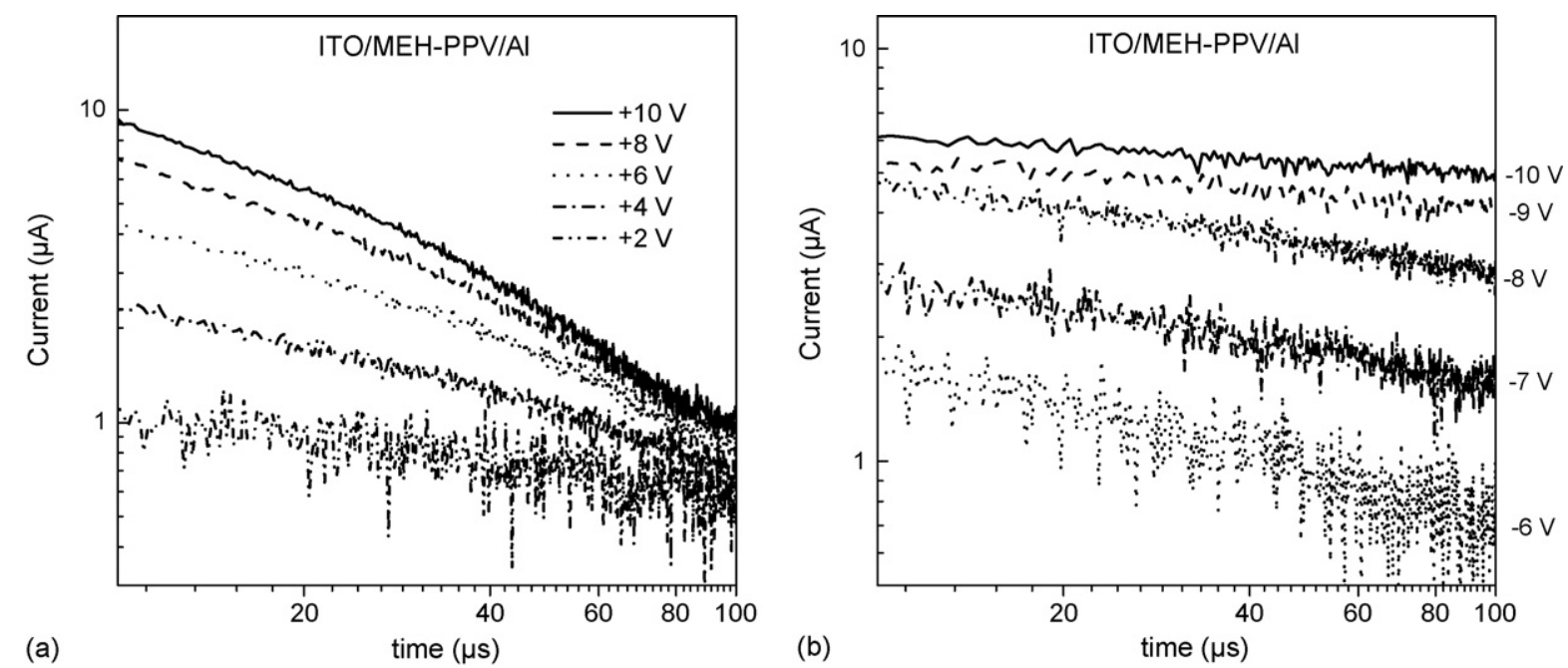

Fig. 5. Transient photoconductivity transits for the ITO/MEH-PPV/Al device at $300 \mathrm{~K}$ : (a) current dominated by holes and (b) current dominated by electrons.

high positive voltages are applied, the built-in voltage is surmounted by the external bias and the observed symbatic behavior is simply explained considering a model in which directly carrier photogeneration (or weakly bound pairs) occurs. An analog explanation can be used to explain the photocurrent spectrum of the ITO/MEH-PPV/Au device.

\section{Conclusions}

Photocurrent action spectra were used to observe the existence of built-in voltages in polymeric LEDs which are strongly dependent on the work-function of the metals used as electrodes. The open-circuit voltages observed in the $I-V$ curves under illumination can be used to determine, for each device, the built-in voltage. Transient current measurements were used to confirm that the mobility of positive photogenerated free charges is much higher than the mobility of the negative ones. This information is valuable in order to explain qualitatively the observed photocurrent spectra behavior. A more detailed semi-quantitative model is being developed to be published soon elsewhere.

\section{References}

[1] R.N. Marks, J.J.M. Halls, D.D.C. Bradley, R.H. Friend, A.B. Holmes, J. Phys. Condens. Matter. 6 (1994) 1379-1394.

[2] C.H. Lee, G. Yu, A.J. Heeger, Phys. Rev. B 47 (1993) 1554315553.

[3] X. Wei, M. Raikh, Z.V. Vardeny, Y. Yang, D. Moses, Phys. Rev. B 49 (1994) $17480-17483$.

[4] D. Moses, H. Okumoto, D. Comoretto, C.H. Lee, A.J. Heeger, T. Ohnishi, T. Nogushi, Synth. Met. 84 (1997) 539-544.

[5] D. Moses, D. Comoretto, C.H. Lee, A.J. Heeger, Synth. Met. 84 (1997) $559-562$.

[6] M.G. Harrison, J. Grüner, G.C.W. Spencer, Phys. Rev. B 55 (1997) 7831-7849.

[7] N. Chawdhurry, A. Köhler, M.G. Harrison, D.H. Hwang, A.B. Holmes, R.H. Friend, Synth. Met. 102 (1999) 871-872.

[8] M. Gailberger, B.N. Thanh, H. Bässler, Mol. Cryst. Liq. Cryst. 217 (1992) $217-221$.

[9] F. Wudl, G. Srdanov, US Patent No. 5189136, 233 (1993).

[10] H.B. DeVore, Phys. Rev. 102 (1956) 86-91.

[11] D.M. Pai, J. Chem. Phys. 52 (1970) 2285-2291. 